\title{
Smart Agri-Food Logistics: Requirements for the Future Internet
}

\author{
C.N. Verdouw ${ }^{1}$, H. Sundmaeker ${ }^{2}$, F. Meyer ${ }^{2}$, J. Wolfert $^{1}$, J. Verhoosel ${ }^{3}$ \\ ${ }^{1}$ LEI, part of Wageningen UR, The Hague, The Netherlands; \\ ${ }^{2}$ ATB Institut für Angewandte Systemtechnik Bremen GmbH, Bremen, Germany; \\ ${ }^{3}$ TNO, Delft; The Netherlands
}

\begin{abstract}
The food and agribusiness is an important sector in European logistics with a share in the EU road transport of about $20 \%$. One of the main logistic challenges in this sector is to deal with the high dynamics and uncertainty in supply and demand. This paper defines requirements on Future Internet (FI) technologies that have to be met to accomplish the specific challenges of agri-food logistics. It identifies a set of generic technical enablers as input for the realisation of a FI core platform. This technology foundation is to be developed and tested in a Future Internet public-private partnership (FI-PPP) environment of over 150 organisations.
\end{abstract}

\section{Introduction}

Agri-food is an important sector in European logistics. The agri-food logistics covers $19 \%$ of the transports within the EU and $25 \%$ of the international EU transports [1]. One of the main logistic challenges for the agri-food industry is to deal with high dynamics and uncertainty in supply and demand [2,3]. There is great uncertainty regarding fresh product quality as well as available volumes in time on a specific place. The sector is characterised by last-minute changes and rushorders. As a consequence, the required prediction and planning concept and accompanying logistics system needs to be very flexible, enabling last minutes changes and reallocations, but also provide a robust planning [2-5].

In this paper it will be argued that current internet limitations are important bottlenecks to accomplish the specific challenges of agri-food logistics. Important limitations that are addressed by the EC's FI Architecture Group include i) a lack of data integrity, reliability, provenance and trust; ii) a lack of data integration and federated storage solutions; iii) lack of flexibility and adaptive control; and iv) segmentation of data and control [6].

The intended development of Future Internet (FI) technologies is promising to overcome these limitations. The FI is a general term that labels the emergence of a 
new era in the evolution of the Internet. It combines several trends in internet development into an integrated approach. These trends include [7]:

- the on-going industrialization of IT in the form of cloud computing and open service delivery platforms;

- new wireless networking technologies and the deployment of fibre that are paving the way for new (real-time) applications;

- the breakthrough of the Internet of Things, with the vision of ubiquitously connecting intelligent devices and sensors.

The objective of the research presented in this paper is to define the requirements on the Future Internet (FI) technologies of the food and agribusiness domain. Therefore, after an introduction of the method, the remainder of this paper first describes the logistic challenges in the agri-food sector and the envisaged FI-based solution. Next, an analysis of user requirements for FI logistics is presented.

\section{Method}

\subsection{Future Internet PPP and the Smart Agri-Food project}

The research presented in this paper is carried out as a part of the SmartAgri-Food project ("Future Internet for Safe and Healthy Food from Farm to Fork") within the ICT theme of the FP7 (www.smartagrifood.eu). SmartAgriFood is one of eight currently being funded users case studies of the "Future Internet Public Private Partnership" programme (FI-PPP, www.fi-ppp.eu).

The FI-PPP programme aims to advance Europe's competitiveness in FI technologies and systems and to support the emergence of FI-enhanced applications of public and social relevance. This will be achieved by (1) development of a smart, Europe-wide internet-powered infrastructure, (2) increasing the suitability for processing larger amounts of data, (3) ensuring a more secure and reliable use of Internet, allowing real-time data processing for real-time applications. In technical terms the FI-PPP targets a versatile and open network and service platform, supported by reusable, standardised and commonly shared technology enablers serving a multiplicity of use cases in different sectors [8].

The FI-PPP programme currently includes over 150 partners. It follows a userdriven approach, in which 8 use case projects define the requirements for the realisation of a FI core platform. Subsequently, this technical foundation of the FI is to be developed and tested in its public-private partnership environment. 
The SmartAgriFood project addresses the food and agribusiness as a use case for the FI. It aims to boost the application and use of FI ICTs in the agri-food sector by identifying and describing/ developing:

- technical, functional and non-functional FI-specifications for experimentation in the food and agribusiness;

- smart agri-food-specific capabilities and conceptual prototypes, demonstrating critical technological solutions including the feasibility to further develop them in large scale experimentation and validation;

- existing experimentation structures and start user community building, resulting in an implementation plan for the next phase of the FI-PPP programme.

\subsection{Methodology}

The Smart Agri-Food project is focussing on three sub systems of the sector: Smart Farming, Smart Agri-Food Logistics and Smart Food Awareness (consumer). This paper presents the results of the definition for experimentation of the second sub system: Smart Agri-Food Logistics. Therefore, seven specific application scenarios have been identified and a template for the investigation of the application was developed. Experts from academia, non-profit research institutes, ICT industry and food industry were analysing the scenarios to identify ICT related aspects and to derive specific user related functional requirements. The requirements were clustered into functional blocks based on i) a logical association of the different functional requirements that address similar tasks, and ii) the socalled generic enablers that shall be provided by the FI Core Platform [7, 9].

\section{Smart Logistics in the Agri-Food sector}

\subsection{Specific logistic challenges in agri-food supply chains}

The sector-specific characteristics of the food and agribusiness heavily impact logistics in this sector [2-5]:

- High supply uncertainty due to natural production: unpredictable variations in quality and quantity of supply, which demands for flexibility in logistic processes and planning and early warning and pro-active control mechanisms; 
- High perishability of fresh food products, which demand for temperatureconditioned transportation and storage (cold chains) and very short order-todelivery lead-times;

- Seasonable growing, which demands for global sourcing to ensure year-round availability;

- High demands on food safety, quality and (environmental) legislation, which demands for the ability to trace production information of products in transit;

- High flow complexities, due to a combination of continuous and discrete product flows, diverging and converging processes and by-products; this demands for advanced tracking and tracing and logistic planning capabilities;

- Important role of import/export, including additional phytosanitary and veterinary inspections;

- Complex network structure where small and medium enterprises trade with huge multinationals in the input and retail sector; this demands for proper collection and regional orchestration in logistic mainports and proper allocation mechanisms to connect aggregated demand with fragmented supply.

Due to these characteristics the application of existing ICT solutions is not always obvious and less straightforward than it might be in other industries. For example: there is a high need for interoperability but this should be accompanied with flexibility to deal with the high dynamics in agri-food logistics. Furthermore, the need for sector-specific ICT solutions is high, but at the same time the possibilities for investments are low due to the large number of SMEs. Consequently, the current state of the art of ICT in the agri-food logistics is characterized by large amounts of available data, but there is a poor level of integration and the support for intelligent use of these data is insufficient. The complexity of current solutions is too high and jeopardizes the development and operation of affordable solutions. As a result, the adoption of internet for basic information services is high, but the use for more advanced functionalities is limited.

These complexities contrast with the high and constantly increasing need for better information, control and transparency in the agri-food sector. Consequently, there is a mismatch between the state of information technology in agri-food and the high and increasing need for intelligent solutions that combine interoperability with flexibility and that are both sector-specific and suitable for SMEs. The next section introduces a vision on how to deal with these challenges.

\subsection{Smart Agri-Food Logistics enabled by the Future Internet}

The envisaged solutions focus on the enhancement of new types of efficient and responsive logistics networks with flexible chain-encompassing tracking and tracing systems and decision support based on that information. These systems effectively virtualise the logistics flows from farm to fork, support a timely and error- 
free exchange of logistics information and provide functionality for intelligent analysis and reporting of exchanged data to enable early warning and advanced forecasting. Three critical features of these systems are distinguished: real-time virtualization, logistics connectivity and logistics intelligence (see Figure 1).

As argued in the previous section, current solutions do not sufficiently provide these features. The FI offers a realistic scenario to solve this because it aims at a provision of an integrated architecture that overcomes the basic limitations of cur-

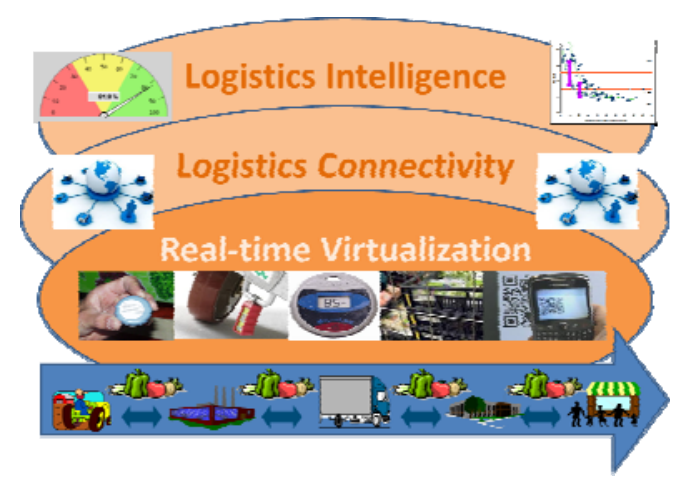

Figure 1 Smart Agri-Food Logistics rent information systems for agri-food logistics.

The envisaged Smart Agri-Food Logistics solutions will build on the Core Platform that is being developed by the FI-PPP programme [7-9]. This platform aims at a provision of an innovative infrastructure for cost-effective creation and delivery of versatile digital services, providing high Quality of Service and security guarantees. The basic underlying approach is that the Core Platform will offer reusable and commonly shared capabilities and functionalities (Generic Enablers) which can be flexibly customized, used and combined for many different Usage Areas (pick, plug and play). The FI Core Platform will contain Generic Enablers for Cloud Hosting, Data/Context Management, Applications/Services Ecosystem and Delivery Framework, Internet of Things (IoT) Services Enablement, Interface to Networks and Devices (I2ND) and Security $[7,9]$.

Products implementing these Generic Enablers can be picked and plugged together with complementary products (Specific Enablers) in order to build domainspecific instances [7]. Smart Agri-Food Logistics is intended to be one of those instances (as part of the Smart Agri-Food project). The next section elaborates on the specific requirements that should be met for that purpose.

\section{Requirements on the Future Internet}

\subsection{Basic Future Internet challenges in Agri-Food Logistics}

Smart Agri-Food Logistics imposes specific demands on the development of FI technologies. The identified basic challenges are : 
- Internet of Things (IoT): Various types of sensors (e.g. temperature loggers) are used in the agri-food logistics sector. These applications are mainly limited in scope and focus on specific subparts of the logistics chain. There are communication breaks, especially when transport units or ownership is changed. Communication aspects of the IoT are already fairly well covered by existing technologies, such as networked RFID, wireless sensor networks and near-field communication. A FI is expected to enable a capturing at an integrated global sensor information level in which combinations of sensor information can be used to constantly monitor the product status and enable the identification of exceptions or deviations from expected conditions from a chain perspective.

- Telematics Systems: To promptly react to changes of ambient parameters (e.g. temperature, light, ethylene concentration, etc.), the sensors are already applied in some parts of agri-food logistics. When merging this with further information about location, speed and context a new dimension of forecasting and reactions will be possible. However, dynamic change of interrelationships of food chain partners is imposing a key challenge for integrating different systems and communicating information. There is a need for an advanced decentralised management of authentication, authorisation and revocation of access rights for enabling advanced governance models while assuring privacy of data.

- Tracking and Tracing: Well-known services are in place, but often limited to an internal level of traceability or to an external approach by service providers. If we increase the level of traceability and focus on the whole supply-chain, the system needs to be able to trace across and through the companies in the chain. However, there are some holistic approaches available but generally need to apply a central entity that is compiling all the information. At the same time the food chain actors are reluctant to provide all data to such a central entity.

- Autonomous Systems: Most available logistics systems implement a strict centralized approach and manage logistic processes in a top-down way. Opposite to centralised systems, decentralised systems are trying to translate the current predominant hierarchy approach into a heterarchy model. To achieve this, each entity inside the logistics processes is able to process information, take decisions autonomously and communicate with other entities. Similar to the IoT topic, these capabilities implicate the existence of a virtual identity representing the physical one, but encapsulating the intelligence and ensuring the integrity of content and certifying "mobile software components" to be used on different systems operated by the actors in the food chain.

- Business Intelligence: To support strategic and functional decisions, BI systems analyse company-, competitors- and market-relevant electronic accessible data and process models by acquisition, processing and dissemination. This domain can be considered as a beneficiary of the technologies discussed before. Hence, as soon as the exploitation of the other ICTs will advance, tremendous improvements of BI seem possible. 
In addition to these technological challenges, it became also evident that the realisation of a FI cannot be accomplished purely on a technology-driven basis. Organisational conditions are crucial success factors, in particular: a proper business case for all involved participants, agreement on the distribution of benefits, trust in the privacy of data and a business process orientation.

\subsection{Specific application scenarios}

Specific application scenarios are elaborated as kind of test beds to prepare a larger scale experimentation with respect to FI potentials for the agri-food logistics. Those application scenarios are defined based on the type of logistics information. Distinction is made between identification, static and dynamic state attributes, including condition parameters, product specifications and compliance information. Based on this classification the following table 1 is identifying the product categories and user groups that are mainly involved.

Table 1. Main involved users of the application scenarios

\begin{tabular}{|c|c|c|}
\hline Application Scenario & Product Category & Main involved user groups \\
\hline $\begin{array}{l}\text { 1. Intelligent Supply Chain Event } \\
\text { Management systems for the fu- } \\
\text { ture food supply chain }\end{array}$ & Fruit (mango) & Grower, Forwarder, Packer/ Repacker \\
\hline $\begin{array}{l}\text { 2. Exception notification based } \\
\text { on fruits/vegetables chain }\end{array}$ & Fruit \& vegetables & $\begin{array}{l}\text { Farmer, Laboratory, Trader, Trans- } \\
\text { porter, Distribution Centre, Retailer, } \\
\text { Consumer, Service Provider }\end{array}$ \\
\hline $\begin{array}{l}\text { 3. Real-time and trusted infor- } \\
\text { mation regarding product specifi- } \\
\text { cations and compliance }\end{array}$ & Meat & $\begin{array}{l}\text { Primary Producer, Slaughtering/ } \\
\text { Deboning, Processing, Transporter, } \\
\text { Storing, Wholesale, Retail }\end{array}$ \\
\hline $\begin{array}{l}\text { 4. Legal compliance and quality } \\
\text { control }\end{array}$ & $\begin{array}{l}\text { Vegetables (toma- } \\
\text { to) }\end{array}$ & $\begin{array}{l}\text { Producer (or Product Owner), Trans- } \\
\text { porter, Packer, Wholesaler/Trader, } \\
\text { Certification Body, consumer }\end{array}$ \\
\hline $\begin{array}{l}\text { 5. Quality Controlled Logistics } \\
\text { in the flower chain }\end{array}$ & Flowers & Grower, Auction, Trader, Retailer \\
\hline $\begin{array}{l}\text { 6. Intelligent retail store replen- } \\
\text { ishment of fresh products }\end{array}$ & $\begin{array}{l}\text { Fresh produce } \\
\text { (fruits/vegetables) }\end{array}$ & $\begin{array}{l}\text { Retailer (warehouse), Retail Outlet } \\
\text { (supermarkets, hypermarkets, etc.) }\end{array}$ \\
\hline $\begin{array}{l}\text { 7. RFID on pallets from ware- } \\
\text { house to retail store }\end{array}$ & $\begin{array}{l}\text { Fresh products } \\
\text { (such as meat) }\end{array}$ & $\begin{array}{l}\text { Retailer (warehouse), Retail Outlet } \\
\text { (supermarkets, hypermarkets, etc.) }\end{array}$ \\
\hline
\end{tabular}




\subsection{Expert-based definition of user requirements}

The application scenarios are used to define in total 46 FI requirements for smart agri-food logistics. The requirements are grouped into 14 functional blocks. Some $30 \%$ of the requirements are addressing blocks related to data analysis, collection and management, $20 \%$ are addressing the configuration and communication functionalities, while some $15 \%$ are addressing service based architecture and loose coupling principles. Also requirements related to e.g. the IoT, interoperability and immediate user notification are identified.

Subsequently, the requirements are analysed from a more technical perspective and are additionally grouped with respect to envisaged enabling technologies. These functionalities are associating agri-food related aspects, but are serving as trigger to identify and elaborate the envisaged generic enablers that shall be provided by the FI core platform:

- Peer to peer Services: Enabling to map real-life communication inside a supply-chain it should be possible to easily create, join and leave P2P networks. This includes centralised, hybrid and pure P2P networks. Additionally problems like routing of messages through the chain, not requiring a central authority that tracks the latest IP should be addressed, while taking care for routing, prioritisation, message handling and storage.

- Decoupled/Asynchronous transport: To allow working in rural areas and to comply to insufficient network coverage, the overall system should compensate disconnects from the internet on mobile devices by allowing asynchronous communication, enabling to send data without waiting for immediate response.

- Mobile Services/Agents: As indicated before, rural areas not necessarily allow a permanent connection to the internet/cloud. A FI shall enable a transparent user support. This could possibly be supported by migrating services/ agents to user centric devices, since assuring both quality of services and a real time interaction from a user point of view.

- Online profiles: Applications need to fetch information about a given object, like user or machinery profiles. Beyond central or device individual storage, a FI service cloud could also enable the decentralised and distributed storage for a more efficient exploitation of scarce technical resources.

- Updateable profile for objects: Enabling the timely update and/ or synchronisation of especially logistics information (e.g. data for scheduling, notification) that is stored decentralised and distributed by diverse objects in the food chain.

- Entity authentication/authorisation: Mechanism for all types of entities (e.g. actors, objects, etc.) shall be supported, also enabling a decentralised security approach for issuing certificates without a central root. This shall also enable the collaboration in local networks (e.g. devices, machines or ICT supported actors in the field) that are decoupled from the Internet.

- Automatically add/revoke access rights: Automatically adding and/or revoking access rights, based on the changes in the physical as well as in the virtual 
world. This is especially relevant for the physical product flow and related change of e.g. ownership and location along the agri-food chain.

- Identify aggregations of objects: Agri-food logistic objects must be unique identifiable on different levels of aggregation (e.g. cases, pallets, containers). Services for mapping or integration of different unique identification schemes (e.g. GTIN, SSCC, SGLN, GRAI) need to be enabled.

- Virtual Identity: Supporting the generation of virtual identities, while aggregating the identities for objects that are grouped in the physical world, avoiding the need to use active digital objects for each virtual entity (e.g. batch, location, owner related), but also enabling the generation of individual virtual identities.

- Decentralised Trust: Beyond technically trusted relationships, agri-food logistics security systems should allow for human related trusted interventions. This is specifically related to the integration of business process related certification schemes (e.g. Orgainvent). The logistic objects should facilitate the decentralised generation of such human-trusted relationships.

- Mapping of Interfaces: The Internet assures the standardised information exchange based on e.g. http, ftp. There are also divers data exchange standards. However, different legacy systems are offering semantically similar but technical incompatible interfaces. At the same time, they need to import and export information from/to a product related digital/ virtual object, while the interfaces of the different systems remain quite stable. A FI shall support the mapping of those technically different interfaces that can be executed by an experienced user, generally familiar with the semantics.

\section{Conclusions and discussion}

This paper has argued that the FI can be an important enabler of the dynamic logistic systems of the future, as required in the agri-food sector, if it provides specific capabilities. The paper has identified these capabilities. Its objective was to define the requirements on FI technologies that have to be met to accomplish the specific challenges of agri-food logistics.

The paper has first addressed the basic demands for FI logistics concerning Internet of Things (IoT), Telematics Systems, Tracking and Tracing, Autonomous Systems and Business Intelligence. Next, 7 specific application scenarios are used to define 46 specific requirements, grouped into 14 functional blocks. Last, a set of the required technical enablers is identified, i.e. peer to peer services; decoupled/asynchronous transport; mobile services/agents; online profiles; updateable profile for objects; entity authentication/authorisation; automatically add/revoke access rights; identify aggregations of objects; virtual identity; decentralised trust; mapping of interfaces. These generic enablers are provided to the FI-PPP initiative as input for the realisation of a FI core platform. This technology foundation of the 
FI is to be developed and tested in a public-private partnership environment of over 150 organisations.

The main theoretical contribution is that the Smart Agri-Logistics approach utilizes a generic and standardized internet platform to instantiate specific solutions for logistics information systems in the agri-food sector. As a result, it overcomes current bottlenecks and enables the development and operation of affordable solutions that are independent from geographic locations and independent from specific implementation choices. This potentially will boost the application of intelligent information systems for logistics management in agri-food supply chains.

The foremost remaining challenge from a scientific perspective is how to configure dedicated logistic information systems, that provide the right functionality in the specific context of agri-food supply chains, based on a generic internet platform. A complicating factor behind this challenge is that supply chains in the food and agribusiness are characterised by a high variety and variability of process configurations. As a consequence, logistic information systems should enable to take part in different configurations concurrently and to switch quickly to new or adjusted configurations.

The ICT related functionalities, as presented in this paper, will be further considered when specifying the envisaged conceptual prototypes that shall serve for the verification of a large scale implementation. They need to be further coordinated with the envisaged FI core platform in an interactive approach to clearly separate the generic enablers from the envisaged food domain specific enablers.

Acknowledgements - The research leading to these results has received funding from the European Community's Seventh Framework Programme (FP7/2007-2013) under grant agreement $n^{\circ}$ 285326 . The authors greatly acknowledge the involved individuals and companies for their support. In particular our gratitude goes to Michael van Bekkum (TNO), Adrie Beulens (Wageningen University), Yann Cassing (SGS), Marta Fontseré (Bon Preu), Elena Mansilla (SGS), Eloi Montcada (Bon Preu), Joan Sabartés (Bon Preu), Angela Schillings-Schmitz (GS1), Ralph Troeger (GS1), and Nikola Vucic (Huawei).

\section{References}

1 Eurostat/TLN 2008 (data 2007).

2 Verdouw, C.N., Beulens, A.J.M., et al. Mastering demand and supply uncertainty with combined product and process configuration. Int. J. of Comp. Int. Man., 2010. 23(6): 515-528

3 Trienekens, J.H., Wognum, P.M., Beulens, A.J.M. \& van der Vorst, J.G.A.J., 2011. Transparency in complex dynamic food supply chains. Adv. Engineering Informatics, 26 (1), 55-65

4 van Wezel, W., Van Donk, D.P. and Gaalman, G., 2006. The planning flexibility bottleneck in food processing industries. Journal of Operations Management, 24 (3), 287.

5 Verdouw, C.N., Beulens, et al., 2010. Process modelling in demand-driven supply chains: A reference model for the fruit industry. Comp. and Electr. in Agr., 73 (2), 174-187.

6 Zahariadis, T., Papadimitriou, D., et al., Towards a Future Internet Architecture. In: Future Internet Assembly 2011, J. Domingue, et al. (eds.), 2011, Springer, LNCS, 6656, 7-18.

7 Overall FI-WARE Vision. Project Wiki. Date Accessed: 13 January 2011. URL: http://forge.fi-ware.eu/plugins/mediawiki/wiki/fiware/index.php/Overall_FI-WARE_Vision

8 European Commission; Information and Communication Technologies, Work Programme 2011-12; EC; July 2010; http://cordis.europa.eu/fp7/ict/.

9 FI-WARE High-level Description (Product Vision). Version 1.1, 31.08.2011. 